\title{
Fitting SIR model to COVID-19 pandemic data and comparative forecasting with machine learning
}

\author{
Mouhamadou A.M.T. Baldé \\ University of Cheikh Anta Diop. \\ Laboratory of Mathematics of Decision and Numerical Analysis. \\ BP 45087, 10700. Dakar, Senegal. \\ Department of Mathematics of Decision(DMD)-FASEG. \\ mouhamadouamt.balde@ucad.edu.sn
}

\begin{abstract}
In this work, we use a classical SIR model to study COVID-19 pandemic. We aim to deal with the SIR model fitting to COVID-19 data by using different technics and tools. We particularly use two ways: the first one start by fitting the total number of the confirmed cases and the second use a parametric solver tool. Finally a comparative forecasting, machine learning tools, is given.
\end{abstract}

Keywords - fitting, COVID-19, SIR model scaling, SIR model fitting, predict, machine learning

\section{INTRODUCTION}

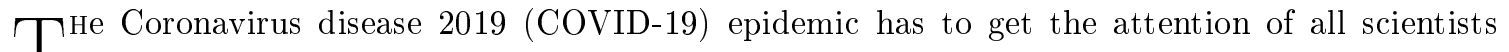
1 around the world. The COVID-19 appears in November 2019 in Wuhan, central China. In March 2020, the epidemic was reclassified as a pandemic by the World Health Organization (WHO). The COVID-19 pandemic is spreading rapidly in many other countries. Measures, limiting humanto-human contact (social distancing, barrier measures, confinement), are taken to stem the spread, causing a sudden slowdown in the world economy and a stock market crash on March 12, 2020. The COVID-19 is contagious with human-to-human transmission. The incubation period is generally between two and fourteen days, with an average of five days.

Many researchers have used new or classical models of infectious diseases to describe, study or predict the evolution of COVID-19 pandemic.

In this work, we aim to use the well known classical SIR model Anderson and May, 1991], [Hethcote, 2000, to fit it to world data of COVID-19. We discuss and apply different methods and show numerical results. The methods consist in first to use the work in Liu, Magal, Seydi and Webb, 2020 which start by fitting the total number of the confirmed cases and in second to use parametric solver.

SIR stands for "Susceptible-Infected-Removed". If we consider that all removed individuals are the recovered individuals, then we can say "Susceptible-Infected-Recovered".

In this paper, we first introduce the classical mathematical SIR models we use to fit COVID-19 data. In the second we present different mechanisms we use to fit the data. Then we show numerical results. And finally, we show some prediction for Senegal of the SIR models we study compared with forecasting using machine learning. 


\section{CLASSICAL SIR MODEL}

The classical SIR model is given as follow:

$$
\left\{\begin{array}{l}
\frac{d S}{d t}=-\frac{\beta S(t) I(t)}{N} \\
\frac{d I}{d t}=\frac{\beta S(t) I(t)}{N}-\gamma I(t) \\
\frac{d R}{d t}=\gamma I(t)
\end{array}\right.
$$

with the dependent variables $S(t), I(t), R(t)$ being respectively the number of susceptible individuals at time $t$, the number of infectious individuals at time $t$ and the number of recovered individuals at time $t$. In addition, the unknowns $S(t), I(t), R(t)$ satisfy $N=S(t)+I(t)+R(t) . N$ is the total number of individuals.

The initial conditions are $S(0)=S_{0} \geq 0, I(0)=I_{0} \geq 0$ and $R(0)=R_{0} \geq 0$.

Some parameters must be presented: $\beta$ is the contact rate, $1 / \gamma$ is the average infectious period, $\mathcal{R}_{0}=\beta / \gamma$ is the basic reproduction number.

The SIR model satisfies some properties:

- The susceptible function $S(t)$ decreases to $S_{\infty}=S_{0} \exp \left(-\frac{\mathcal{R}_{0}}{N}\left(R_{\infty}-R_{0}\right)\right)$ as $t \rightarrow \infty$.

- The recovered function $R(t)$ increases up to $R_{\infty}=N-S_{0} \exp \left(-\frac{\mathcal{R}_{0}}{N}\left(R_{\infty}-R_{0}\right)\right)$ as $t \rightarrow \infty$.

- If $\mathcal{R}_{0}>N / S_{0}$, then $I(t)$ increases up to a maximum value $I_{\text {max }}=I_{0}+S_{0}-\left(1+\ln \left(\frac{\mathcal{R}_{0} S_{0}}{N}\right)\right) \frac{N}{\mathcal{R}_{0}}$.

- If $\mathcal{R}_{0}<N / S_{0}$, then $I(t)$ decreases to 0 .

Remark II.1. At the outset of an epidemic, nearly everyone (except the infected case) is susceptible. So we can say that $S_{0} \approx N$ and then we can replace $N / S_{0}$ by 1 .

It is also possible to couple the SIR model with other differential equations like death equation to study the number of death due the epidemic disease or economic model equations to study the impact of the epidemic disease on the economy. To couple the SIR model with a death equation, we consider the death rate $\mu$ due to the infection. So the system is given by:

$$
\left\{\begin{array}{l}
\frac{d S}{d t}=-\frac{\beta S(t) I(t)}{N} \\
\frac{d I}{d t}=\frac{\beta S(t) I(t)}{N}-\gamma I(t)-\mu I(t) \\
\frac{d R}{d t}=\gamma I(t) \\
\frac{d D}{d t}=\mu I(t)
\end{array}\right.
$$

with $D$ being the number of death due to the infection. Then the total removed is $R(t)+D(t)$ and $N=S(t)+I(t)+R(t)+D(t)$. 


\section{METHODS}

\section{i. Fitting function to data}

The starting point of the work is to find a function that fits the data of total confirmed cases. As in Liu, Magal, Seydi and Webb, 2020, we set the total number of infectious cases by:

$$
T N I(t)=\int_{t 0}^{t} I(s) d s .
$$

The total number of infectious cases function, that fit the data, can be written as $T N I(t)=$ $b \exp (c t)-a$. Where $a, b, c$ are parameters to estimate by using the least square method. Then with $T N I(t)$ we can obtain information on the unknown functional variables and parameters of the SIR model.

After calculation as in [Liu, Magal, Seydi and Webb, 2020, we obtain:

- $t_{0}=\frac{\ln (a)-\ln (b)}{c}$.

- $I(t)=I\left(t_{0}\right) \exp \left(c\left(t-t_{0}\right)\right)$, with $I\left(t_{0}\right)=I_{0}=a c$.

- $R(t)=\frac{\gamma\left(I(t)-I_{0}\right)}{c}$.

- $\beta=N \frac{c+\gamma}{S_{0}}$.

The simulation results are given in subsection is figure $1 \mathrm{a}, 1 \mathrm{~b}, 1 \mathrm{c}$ and $1 \mathrm{~d}$, Also the values of the parameters $\beta$ and $\gamma$ are given.

We consider now that at a time $T$ some measures are taken like social distancing, half or full confinement. It can be interpreted as the contact rate is reduced by some factor or at a time point, the contact rate is close to 0. Indeed in Liu, Magal, Seydi and Webb, 2020, the authors considered that the transmission of COVID-19 from infectious to susceptible individuals stopped after strong measures has been taken in China. Then they have fixed the contact rate to 0 . In [Lauro, Kiss and Miller, 2020, the authors considered that the contact rate is reduced by some factor when a social distancing intervention is introduced with a certain duration. Then they have replaced $\beta$ by $(1-c) \beta$. It is also possible to consider that $\beta$ will decrease depending on time like in Liu, Magal, Seydi and Webb, 2020, where authors has considered an exponential decreasing function of time.

In this work, we consider that the contact rate decreases progressively to 0 . Then we choose continuous function with a slow decrease to describe the contact rate starting at the date-time of the measures.

We propose two types of function for $\beta$ noted $\tilde{\beta}(t)$.

The first one is :

$$
\tilde{\beta}(t)= \begin{cases}\beta & \text { if } t \in\left[t_{0}, T\right] \\ \beta\left(\frac{T}{t}\right)^{\delta / p} & \text { if } t>T,\end{cases}
$$

where $\delta$ and $p$ are parameters to choose. The second one is:

$$
\tilde{\beta}(t)= \begin{cases}\beta & \text { if } t \in\left[t_{0}, T\right] \\ \beta-\frac{\left(\ln \left(\frac{T}{t}\right)\right)^{\kappa}}{q} & \text { if } t>T,\end{cases}
$$

where $\kappa$ and $q$ are parameters to choose. 
Then replacing $\beta$ by $\tilde{\beta}(t)$ in the SIR model 1 , we obtain:

$$
\left\{\begin{array}{l}
\frac{d S}{d t}=-\frac{\tilde{\beta}(t) S(t) I(t)}{N} \\
\frac{d I}{d t}=\frac{\tilde{\beta}(t) S(t) I(t)}{N}-\gamma I(t) \\
\frac{d R}{d t}=\gamma I(t)
\end{array}\right.
$$

By solving (6), we obtain the new expressions of $T N I(t), I(t)$ and $R(t)$. We show the results in subsection i.

Remark III.1. 1. The values of the parameters $\delta, p, \kappa$ and $q$ can be fixed such that $\tilde{\beta}(t)$ decreases slowly.

2. Since $\tilde{\beta}$ depends on time, we must always consider values of $t$ such that $\tilde{\beta}$ remains positive.

3. $\tilde{\beta}$ given by 4 is positive for all time while $\tilde{\beta}$ given by 5 is positive only if $t \in\left[0, T \exp \left((\beta q)^{1 / \kappa}\right)\right]$.

\section{ii. Fit to data with scaling}

When there is not enough data the fit is generally difficult since the maximal values of the dependent variables can be very huge. For example, $S_{0}$ depends on the total population and generally $S_{0} \approx N$, with $N$ the size of a chosen sample of the population. In SIR models, it is generally possible to choose different population sizes with similar characteristics. If we have characteristic measures we can make the model dimensionless so that the quality of the results is always good. Then we scale the SIR model to the data by using scaling [Langtangen and Pedersen, 2016.

Let us fix the following constant characteristics size: $t_{c}, S_{c}, I_{c}, R_{c}$ respectively of the time $t$, the susceptible $S(t)$, the infected $I(t)$ and the recovered $R(t)$. Then the dimensionless variables $\bar{t}, \bar{S}, \bar{I}, \bar{R}$ are given by $t=\bar{t} t_{c}, S=\bar{S} S_{c}, I=\bar{I} I_{c}, R=\bar{R} R_{c}$.

Replacing in (1) and calculating, we obtain the scaled system:

$$
\left\{\begin{array}{l}
\frac{d \bar{S}}{d t}=-\frac{\beta I_{c} t_{c}}{N} \bar{S}(t) \bar{I}(t) \\
\frac{d \bar{I}}{d t}=\frac{\beta S_{c} t_{c}}{N} \bar{S}(t) \bar{I}(t)-\gamma t_{c} \bar{I}(t) \\
\frac{d \bar{R}}{d t}=\gamma \frac{I_{c} t_{c}}{R_{c}} \bar{I}(t)
\end{array}\right.
$$

Remark III.2. For the adjusting of the data, we choose $t_{c}=t_{\text {max }}^{d}$, with $t_{\text {max }}^{d}$ being the last time of the data. 
The scaled system can be written, by dropping the "-", as follows:

$$
\left\{\begin{array}{l}
\frac{d S}{d t}=-\tilde{\beta}_{1} S(t) I(t) \\
\frac{d I}{d t}=\tilde{\beta}_{2} S(t) I(t)-\tilde{\gamma}_{1} I(t) \\
\frac{d R}{d t}=\tilde{\gamma}_{2} I(t)
\end{array}\right.
$$

with $\tilde{\beta}_{1}=\frac{\beta I_{c} t_{c}}{N}, \tilde{\beta}_{2}=\frac{\beta S_{c} t_{c}}{N}, \tilde{\gamma}_{1}=\gamma t_{c}$ and $\tilde{\gamma}_{2}=\gamma \frac{I_{c} t_{c}}{R_{c}}$.

Remark III.3. By choosing the characteristics such that $S_{c}=I_{c}=R_{c}$, we obtain $\tilde{\beta}_{1}=\tilde{\beta}_{2}$ and $\tilde{\gamma}_{1}=\tilde{\gamma}_{2}$. And then we get a new SIR model.

\section{iii. Parametric solve}

We estimate the parameters $\beta$ and $\gamma$ by using parametric solver in Mathematica. With the parametric solver, we solve the SIR model (2) with solutions depending on the parameters we want to estimate such that the model fits the data. Then plotting the parametric solution for different values of $\beta$ and $\gamma$, finally, give a fit. Parametric solver typically solve differential equations by going through several different stages, depending on the type of equations.

\section{iv. Machine learning}

Machine learning is programming computers to optimize a performance criterion using example data or past experience. We have a model defined up to some parameters, and learning is the execution of a computer program to optimize the parameters of the model using the training data or past experience. The model may be predictive to make predictions in the future, or descriptive to gain knowledge from data or both.

Machine learning uses the theory of statistics in building mathematical models because the core task is making inferences from a sample.(See Alpaydin, 2010).

Then machine learning can be used for the estimation of parameters of an epidemic model. We can go deep by doing data mining since we have massive world data. We can use machine learning to learn about the worldwide data of pandemic COVID-19 and then to predict the future evolution of the disease.

We can also try to learn on the spatial distribution and progression of the disease and predict the location susceptible to be a high level of risk.

In this work, we just use a machine learning tool to learn on small data of Senegal's COVID-19 cases and then to predict the evolution in future days. We use it as a comparison with the other forecasts based on the work we do in this paper.

\section{Numerical Simulations}

\section{i. The data}

In this subsection we show table of data we use for simulation. The tables 1 , 2 and 3 give data respectively of Senegal, China and France. Data for Senegal is obtained from daily press releases on 
SIR model fit to COVID-19 data 2020 , April $\bullet$ Vol. , No.

the COVID-19 from the Ministry of Health and Social Action (http://www.sante.gouv.sn/) and the data for China and France come from [Wolfram Research]. [Wolfram Research provide estimated confirmed COVID-19 infection trend by country or region, based on WHO, U.S. CDC, ECDC, China CDC (CCDC), NHC and DXY. The source dataset is compiled daily by Johns Hopkins CSSE. For details see: https://github.com/CSSEGISandData/COVID-19/issues.

Table 1: COVID-19 data for Senegal: 2020 march 02-31.

\begin{tabular}{|c|c|c|c|c|c|c|c|c|c|c|c|c|c|c|c|c|c|c|c|c|c|c|}
\hline Date & 2345678910 & 11 & 12 & 213 & 14 & 15 & 16 & 17 & 18 & 19 & 20 & 21 & 22 & 23 & 24 & 25 & 26 & 27 & 28 & 29 & 30 & 31 \\
\hline New cases & \begin{tabular}{|lllllllll}
1 & 1 & 1 & 1 & 0 & 0 & 0 & 0 & 0
\end{tabular} & 1 & 5 & 11 & & 2 & 1 & 4 & 5 & 2 & 9 & 9 & 11 & 12 & 7 & 13 & 6 & 14 & 11 & 12 & 20 & 13 \\
\hline Total cases & 1223444444 & 5 & 10 & 21 & 24 & 26 & 27 & 31 & 36 & 38 & 47 & 56 & 67 & 79 & 986 & 99 & 105 & 119 & 130 & 142 & 162 & 175 \\
\hline Recovered cases & $: \begin{array}{lllllllll}0 & 0 & 0 & 0 & 1 & 1 & 1 & 1 & 1\end{array}$ & 1 & 2 & 2 & 2 & 2 & 2 & 2 & 2 & 5 & 5 & 5 & 5 & 8 & 8 & 9 & 9 & 11 & 18 & 27 & 28 & 40 \\
\hline Death cases & $\mid \begin{array}{lllllllll}0 & 0 & 0 & 0 & 0 & 0 & 0 & 0 & 0\end{array}$ & 0 & 0 & 0 & 0 & 0 & 0 & 0 & 0 & 0 & 0 & 0 & 0 & 0 & 0 & 0 & 0 & 0 & 0 & 0 & 0 & 1 \\
\hline
\end{tabular}

Table 2: COVID-19 data for Hubei, China: 2020 January 22- Marsh 31.

\begin{tabular}{|c|c|c|c|c|c|c|c|c|c|c|c|c|c|c|c|c|c|c|}
\hline Date & $\mid 1$ & 2 & 3 & 4 & 5 & 6 & 7 & 8 & 9 & 10 & 11 & 12 & 13 & 14 & 15 & 16 & 17 & 18 \\
\hline Infected cases & 399 & 399 & 494 & 689 & 964 & 1302 & 3349 & 3341 & 4651 & 5461 & 6736 & 10532 & 12722 & 15677 & 18483 & 20677 & 23139 & 24881 \\
\hline Recovered case & s) 28 & 28 & 31 & 32 & 42 & 45 & 80 & 88 & 90 & 141 & 168 & 295 & 386 & 522 & 633 & 817 & 1115 & 1439 \\
\hline Death cases & 17 & 17 & 24 & 40 & 52 & 76 & 125 & 125 & 162 & 204 & 249 & 350 & 414 & 479 & 549 & 618 & 699 & 780 \\
\hline Date & $\mid 19$ & 20 & 21 & 22 & 23 & 24 & 25 & 26 & 27 & 28 & 29 & 30 & 31 & 32 & 33 & 34 & 35 & \\
\hline Infected cases & | 26965 & 28532 & 29659 & 29612 & 43437 & 48175 & 49030 & 49847 & 50338 & 50633 & 49665 & 548510 & 48637 & 46439 & 46395 & 45044 & 43252 & \\
\hline Recovered cases & s| 1795 & 2222 & 2639 & 2686 & 3459 & 4774 & 5623 & 6639 & 7862 & 9128 & 10337 & 71788 & 11881 & 15299 & 15343 & 16748 & 18971 & \\
\hline Death cases & | 871 & 974 & 1068 & 1068 & 1310 & 1457 & 1596 & 1696 & 1789 & 1921 & 2029 & 2144 & 2144 & 2346 & 2346 & 2495 & 2563 & \\
\hline Date & 36 & 37 & 38 & 39 & 40 & 41 & 42 & 43 & 44 & 45 & 46 & 47 & 48 & 49 & 50 & 51 & 52 & \\
\hline Infected cases & | 41603 & 39572 & 36829 & 34617 & 32610 & 30366 & 28174 & 25904 & 23972 & 22628 & 21207 & 719486 & 18247 & 16993 & 15593 & 14407 & 13171 & \\
\hline Recovered case & s| 20969 & 23383 & 26403 & 28993 & 31536 & 33934 & 36208 & 38557 & 40592 & 42033 & 43500 & 45235 & 46488 & 47743 & 49134 & 1 50318 & 51553 & \\
\hline Death cases & 2615 & 2641 & 2682 & 2727 & 2761 & 2803 & 2835 & 2871 & 2902 & 2931 & 2959 & 2986 & 3008 & 3024 & 3046 & 3056 & 3062 & \\
\hline Date & 53 & 54 & 55 & 56 & 57 & 58 & 59 & 60 & 61 & 62 & 63 & 64 & 65 & 66 & 67 & 68 & 69 & 70 \\
\hline Infected cases & $\mid 11755$ & 10421 & 9557 & 8685 & 7751 & 6988 & 6285 & 5715 & 5223 & 4765 & 4317 & 3827 & 3431 & 2895 & 2526 & 2049 & 1726 & 1461 \\
\hline Recovered cases & s) 52960 & 54288 & 55142 & 56003 & 56927 & 57682 & 58382 & 58946 & 59433 & 59882 & 60324 & 60811 & 61201 & 61732 & 62098 & 62570 & 62889 & 63153 \\
\hline Death cases & | 3075 & 3085 & 3099 & 3111 & 3122 & 3130 & 3133 & 3139 & 3144 & 3153 & 3160 & 3163 & 3169 & 3174 & 3177 & 3182 & 3186 & 3187 \\
\hline
\end{tabular}

\section{ii. The function fit}

Here we show simulations related to the subsection i of section III. The formula of $T N I(t)$ is given by $T N I(t)=b \exp (c t)-a$, with $a=13.9324, b=9.61779$ and $c=0.100095$ (figure 1a). We use $\gamma=1 / 7$ and then we obtain: $t_{0}=3.7051, I_{0}=1,39456$. The total population of Senegal is $N=16743927$ from Senegal Population (2020) - Worldometer (www.worldometers.info) and then we obtain $S_{0}=N-I_{0}$. Since $S_{0} \approx N$ we simplify the calculation and obtain $\beta=0,242957$.

Now we consider the time of the measures as 2020, March 23. Then $T=23$. For $\tilde{\beta}$ given by (4), results are shown by figures $2 \mathrm{a}, 2 \mathrm{c}$ and $2 \mathrm{e}$. For $\tilde{\beta}$ given by (5), results are shown by figures $2 \mathrm{~b}, 2 \mathrm{~d}$ 
medRxiv preprint doi: https://doi.org/10.1101/2020.04.26.20081042; this version posted May 1, 2020. The copyright holder for this preprint (which was not certified by peer review) is the author/funder, who has granted medRxiv a license to display the preprint in perpetuity.

It is made available under a CC-BY 4.0 International license.

SIR model fit to COVID-19 data 2020 , April • Vol. , No.

Table 3: COVID-19 data for France: 2020 January 22- Marsh 31.

\begin{tabular}{|c|c|c|c|c|c|c|c|c|c|c|c|c|c|c|c|c|c|c|}
\hline Date & $\mid 1$ & 2 & 3 & 4 & 5 & 6 & 7 & 8 & 9 & 10 & 11 & 12 & 13 & 14 & 15 & 16 & 17 & 18 \\
\hline Infected cases & $\mid 0$ & 0 & 2 & 3 & 3 & 3 & 4 & 5 & 5 & 5 & 6 & 6 & 6 & 6 & 6 & 6 & 6 & 11 \\
\hline Recovered cases & & 0 & 0 & 0 & 0 & 0 & 0 & 0 & 0 & 0 & 0 & 0 & 0 & 0 & 0 & 0 & 0 & 0 \\
\hline Death cases & 0 & 0 & 0 & 0 & 0 & 0 & 0 & 0 & 0 & 0 & 0 & 0 & 0 & 0 & 0 & 0 & 0 & 0 \\
\hline Date & $\mid 19$ & 20 & 21 & 22 & 23 & 24 & 25 & 26 & 27 & 28 & 29 & 30 & 31 & 32 & 33 & 34 & 35 & \\
\hline Infected cases & $\mid 11$ & 11 & 11 & 9 & 9 & 9 & 7 & 7 & 7 & 7 & 7 & 7 & 7 & 7 & 7 & 7 & 2 & \\
\hline Recovered cases & $s \mid 0$ & 0 & 0 & 2 & 2 & 2 & 4 & 4 & 4 & 4 & 4 & 4 & 4 & 4 & 4 & 4 & 11 & \\
\hline Death cases & $\mid 0$ & 0 & 0 & 0 & 0 & 0 & 1 & 1 & 1 & 1 & 1 & 1 & 1 & 1 & 1 & 1 & 1 & \\
\hline Date & $\mid 36$ & 37 & 38 & 39 & 40 & 41 & 42 & 43 & 44 & 45 & 46 & 47 & 48 & 49 & 50 & 51 & 52 & \\
\hline Infected cases & 5 & 25 & 44 & 86 & 116 & 176 & 188 & 269 & 359 & 632 & 926 & 1095 & 1178 & 1739 & 2221 & 2221 & 3570 & \\
\hline Recovered cases & s 11 & 11 & 11 & 12 & 12 & 12 & 12 & 12 & 12 & 12 & 12 & 12 & 12 & 12 & 12 & 12 & 12 & \\
\hline Death cases & 2 & 2 & 2 & 2 & 2 & 3 & 4 & 4 & 6 & 9 & 11 & 19 & 19 & 33 & 48 & 48 & 79 & \\
\hline Date & 53 & 54 & 55 & 56 & 57 & 58 & 59 & 60 & 61 & 62 & 63 & 64 & 65 & 66 & 67 & 68 & 69 & 70 \\
\hline Infected cases & | 4366 & 4396 & 6473 & 7492 & 8883 & 10616 & 12150 & 13708 & 13144 & 16796 & 17923 & 20002 & 22511 & 25269 & 29561 & 30366 & 33599 & 39161 \\
\hline Recovered cases & s 12 & 12 & 12 & 12 & 12 & 12 & 12 & 12 & 2200 & 2200 & 3281 & 3900 & 4948 & 5700 & 5700 & 7202 & 7927 & 9444 \\
\hline Death cases & | 91 & 91 & 148 & 148 & 148 & 243 & 450 & 562 & 674 & 860 & 1100 & 1331 & 1696 & 1995 & 2314 & 2606 & 3024 & 3523 \\
\hline
\end{tabular}

and $2 \mathrm{f}]$

We do the same as in the paragraphs above for France. $T N I(t)=b \exp (c t)-a$, with $a=1, b=0.88$ and $c=0.158$ (figure 3a). We use $\gamma=1 / 7$ and then we obtain: $t_{0}=0.809, I_{0}=0.158$. The total population of France is $N=65241903$ from France Population 2020 (https://worldpopulationreview.com) and then we obtain $S_{0}=N-I_{0}$. Since $S_{0} \approx N$ we simplify the calculation and obtain $\beta=0.300857$.

For France, we consider the time of the measures as 2020, March 17. Then $T=56$. For $\tilde{\beta}$ given by (4), results are shown by figures $4 \mathrm{a}$ and $4 \mathrm{~b}$. For $\tilde{\beta}$ given by (5), results are shown by figures $5 \mathrm{a}$ and $5 \mathrm{~b}$ 


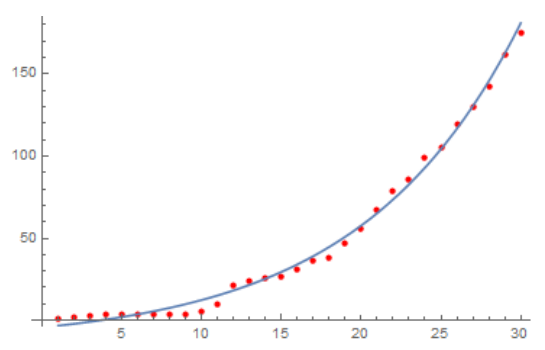

(a) Fit with data of the total cases: $T N I(t)$ is the blue line and the data are the red dotted.

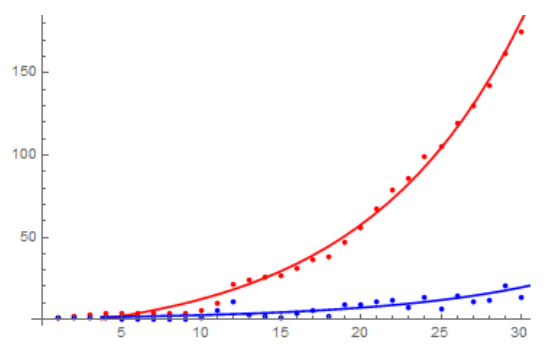

(c) Plot of $T N I(t)$ (red line) with data of total cases(red dotted) and the $I(t)$ (blue line) with the data of new cases(blue dotted).

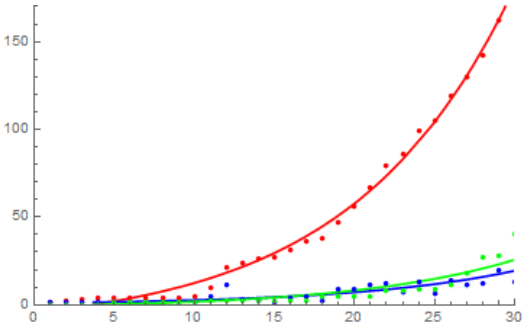

(b) Plot of TNI(t)(red line) with data of total cases(red dotted), the $I(t)$ (blue line) with the data of new cases(blue dotted) and $R(t)$ (green line) with the data recovered cases(green dotted).

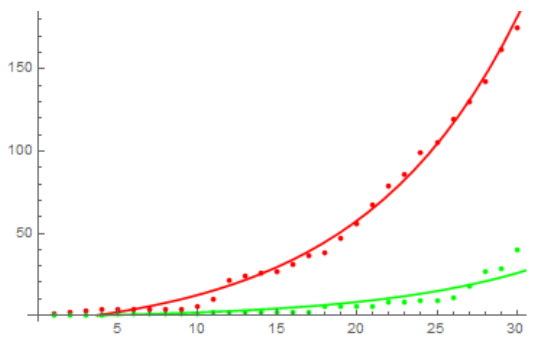

(d) Plot of TNI(t)(red line) with data of total cases(red dotted) and $R(t)$ (green line) with the data recovered cases(green dotted).

Figure 1: Plot of the total number of case data using the SIR model(1) and compared with plot of Senegal's data. On the abscissa axis, the graduation 30 represents 2020, March 31. 


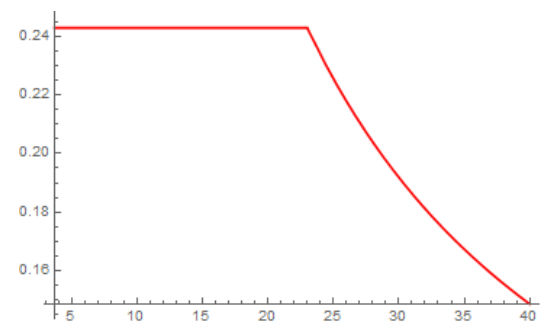

(a) Plot of $\tilde{\beta}(t)$, with $\delta=15$ and $p=10$.

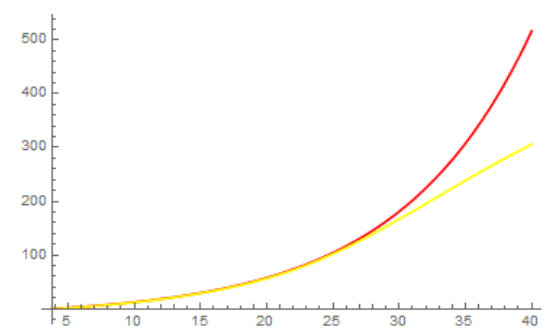

(c) Plot of $T N I(t)$ with $\beta$ (red line) $T N I(t)$ with $\tilde{\beta}$ (yellow line). $\delta=15$ and $p=10$.

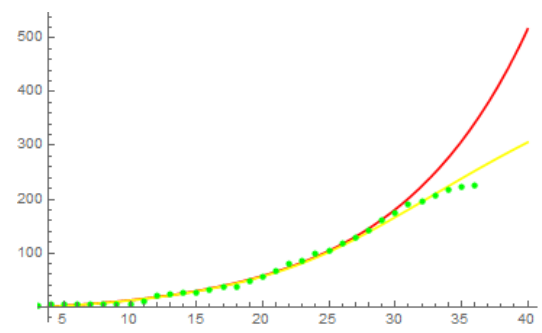

(e) Plot of $T N I(t)$ with $\beta$ (red line), $T N I(t)$ with $\tilde{\beta}$ (yellow line) and data of Senegal . $\delta=15$ and $p=10$.

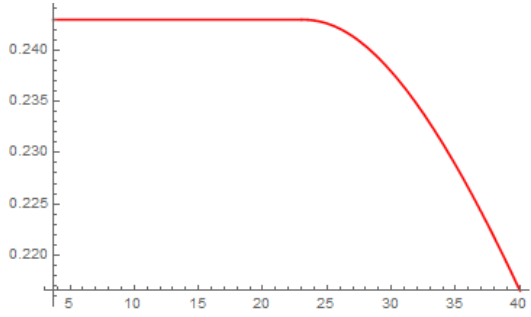

(b) Plot of $\tilde{\beta}(t)$ 5, with $\kappa=1 / 3$ and $q=10$.

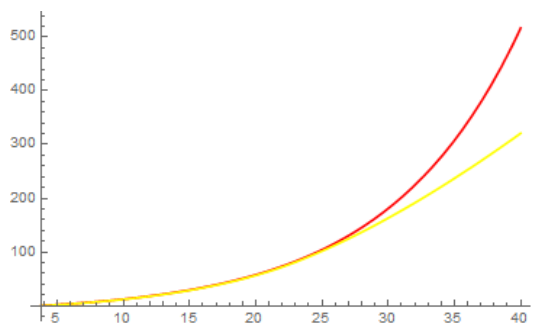

(d) Plot of $T N I(t)$ with $\beta$ (red line) $T N I(t)$ with $\tilde{\beta}$ (yellow line). $\kappa=$ $1 / 3$ and $q=10$.

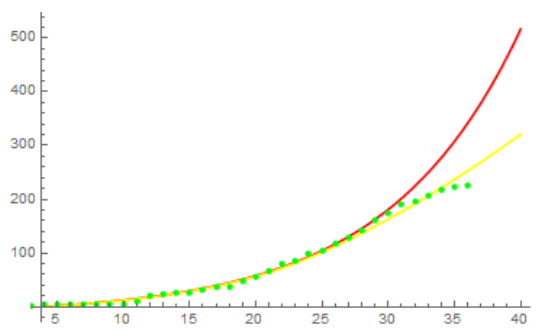

(f) Plot of $T N I(t)$ with $\beta$ (red line), $T N I(t)$ with $\tilde{\beta}$ (yellow line) and data of Senegal. $\kappa=1 / 3$ and $q=10$.

Figure 2: On the top: plot of the contact rate $\tilde{\beta}(t)$ (4) with $\delta=15$ and $p=10$ and $\tilde{\beta}(t)$ (5) with $\kappa=1 / 3$ and $q=10$. In the left for figures related to $\tilde{\beta}(t)$ (4) and in the right for figures related to $\tilde{\beta}(t)$ (5): we plot the two functions TNI $(t)$ compared with plot of Senegal's data in table 1 completed until April 06. On the abscissa axis, the graduation 30 represents 2020, March 31. Then 2020, April 06 is the graduation 36. 


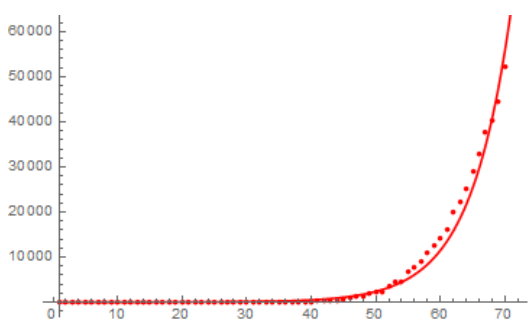

(a) Fit with data of the total cases: $T N I(t)$ is the blue line and the data are the red dotted.

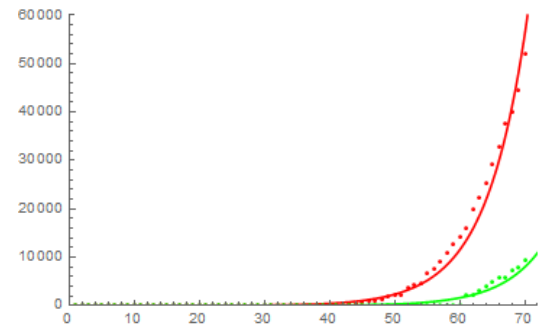

(b) Plot of $T N I(t)$ (red line) with data of total cases(red dotted) and $R(t)$ (green line) with the data recovered cases (green dotted).

Figure 3: Plot of the total number of case data using the SIR model 11 and compared with plot of France's data. On the abscissa axis, the graduation 70 represents 2020, March 31.

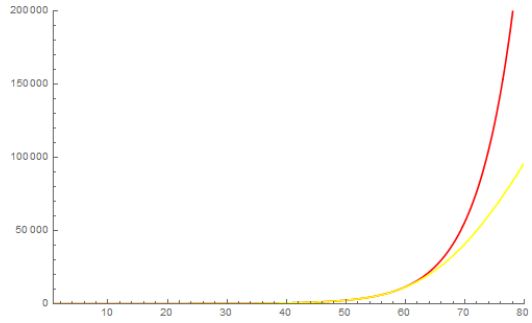

(a) Plot of $T N I(t)$ with $\beta$ (red line) $T N I(t)$ with $\tilde{\beta}$ (yellow line). $\delta=1$ and $p=1 / 2$.

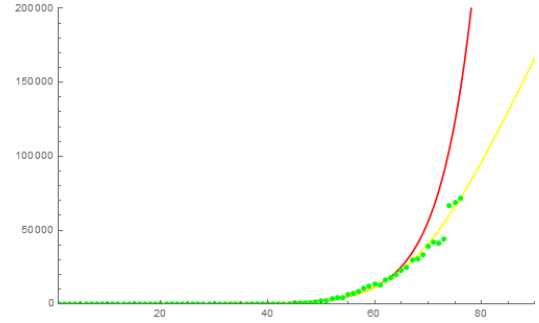

(b) Plot of TNI(t) with $\beta$ (red line), $T N I(t)$ with $\tilde{\beta}$ (yellow line) and data of Senegal. $\delta=1$ and $p=1 / 2$.

Figure 4: Plot of the contact rate $\tilde{\beta}(t)$ with $\delta=1$ and $p=1 / 2$. Plot of the two functions TNI $(t)$ compared with plot of France's data in table 3 completed until April 06. On the abscissa axis, the graduation 70 represents 2020, March 31.

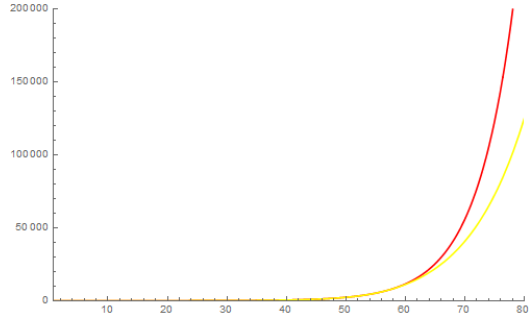

(a) Plot of TNI(t) with $\beta$ (red line) $T N I(t)$ with $\tilde{\beta}$ (yellow line). $\kappa=$ $1 / 4$ and $q=13$.

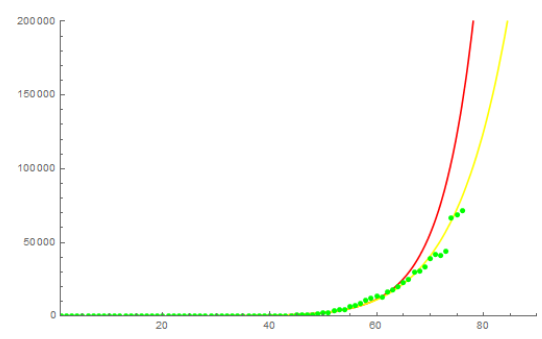

(b) Plot of $T N I(t)$ with $\beta$ (red line), $T N I(t)$ with $\tilde{\beta}$ (yellow line) and data of Senegal . $\kappa=1 / 4$ and $q=13$.

Figure 5: Plot of the contact rate $\tilde{\beta}(t)$ (5) with $\kappa=1 / 4$ and $q=13$. Plot the two functions $T N I(t)$ compared with plot of France's data in table 1 completed until April 06. On the abscissa axis, the graduation 70 represents 2020, March 31. Then 2020, April 06 is the graduation 76. 
medRxiv preprint doi: https://doi.org/10.1101/2020.04.26.20081042; this version posted May 1, 2020. The copyright holder for this preprint (which was not certified by peer review) is the author/funder, who has granted medRxiv a license to display the preprint in perpetuity.

It is made available under a CC-BY 4.0 International license.

SIR model fit to COVID-19 data 2020 , April • Vol. , No.

iii. The fit by scaling

The scaled SIR model 8 is plotted in figure $6 \mathrm{a}$. And in figure $6 \mathrm{~b}$ we fit with the data of Senegal in table 1. We fix the characteristic parameters as: $t_{c}=30$ which is the number of days of the data of Senegal in table 1. $I_{c}=S_{c}=R_{c}=1000$. We use $\beta=5.0753, \gamma=1 / 45$ to obtain $\beta_{1}=\beta_{2}=0.00939349$ and $\gamma_{1}=\gamma_{2}=0.535714$.

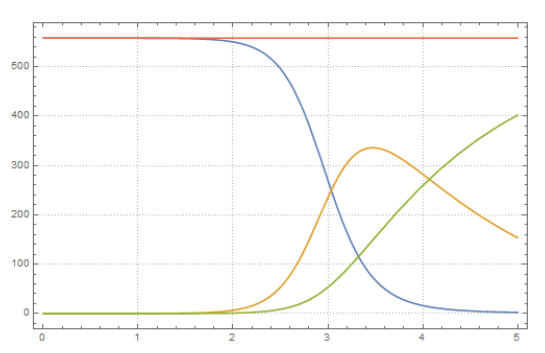

(a) Plot of the scaled SIR model 8 with parameters $t_{c}=30, I_{c}=S_{c}=R_{c}=$ 1000, The susceptible $S(t)$ in blue line, The population in red line, the infected $I(t)$ in orange and the recovered in green line.

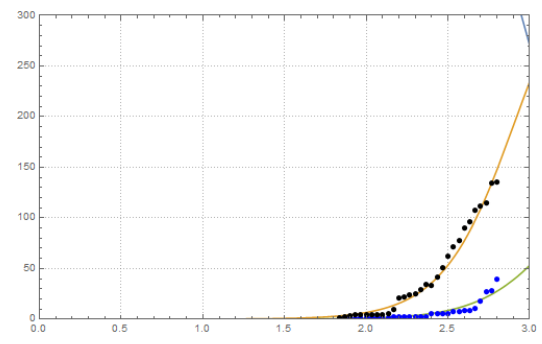

(b) Fit of the infected $I(t)$ in orange to the effective case data in black dotted. And fit of the recovered $R(t)$ in green to the recovered case data in blue dotted. The plotted data is for Senegal. The data is padded 1.8 to the right.

Figure 6: Plot of the scaled SIR model(8) compared with plot of Senegal's data in table 1 . The date 2020 March 31 corresponds to the graduation 2.8 .

\section{iv. The fit by parametric resolution}

Here we use a parametric solver in Wolfram Mathematica to solve the SIR model (2) with respect to parameters $\beta$ and $\gamma$. We start by using the values $\beta=5.0753$ and $\gamma=1 / 45$. Then after we get the new values for the fit. The results are shown in figures $7 \mathrm{a}, 7 \mathrm{~b}$ and $7 \mathrm{c}$ for Senegal, in figures $8 \mathrm{a}$, $8 \mathrm{~b}$ and $8 \mathrm{c}$ for China and in figures $9 \mathrm{a}$, $\mathrm{b}$ and $9 \mathrm{c}$ for France. 
medRxiv preprint doi: https://doi.org/10.1101/2020.04.26.20081042; this version posted May 1, 2020. The copyright holder for this preprint (which was not certified by peer review) is the author/funder, who has granted medRxiv a license to display the preprint in perpetuity.

It is made available under a CC-BY 4.0 International license .

SIR model fit to COVID-19 data 2020 , April $\bullet$ Vol. , No.

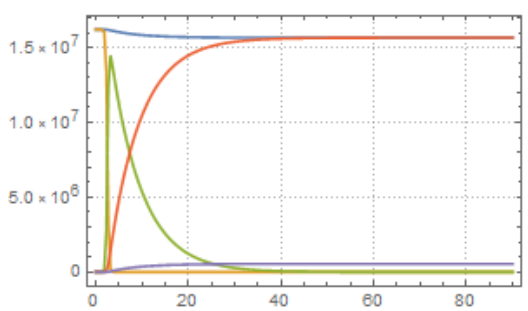

(a) Plot of the SIR model 2 with the parameters $\beta=5.0753$ and $\gamma=1 / 45$ and the proportion of death $\mu=$ $0.01 \gamma$. The susceptible $S(t)$ in red line, The population in blue line, the infected $I(t)$ in green line, the recovered in orange line and the death $D(t)$ in purple.

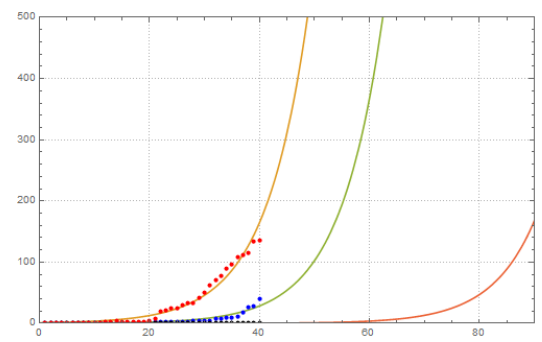

(c) Plot of the SIR model 2 with the parameters $\beta=0.15, \gamma=1 / 45$ and the proportion of death $\mu=0.01 \gamma$. And plot with fit to data.

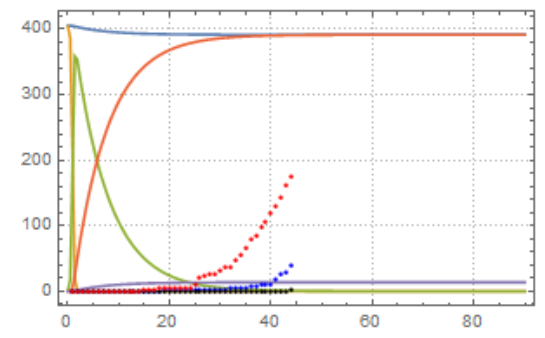

(b) Plot of the SIR model 2 with the parameters $\beta=5.0753, \gamma=1 / 45$, the proportion of death $\mu=0.01 \gamma$ and reduced population. And plot without fit to the Infected data in red dotted, the recovered data in blue dotted, the death data in black dotted.

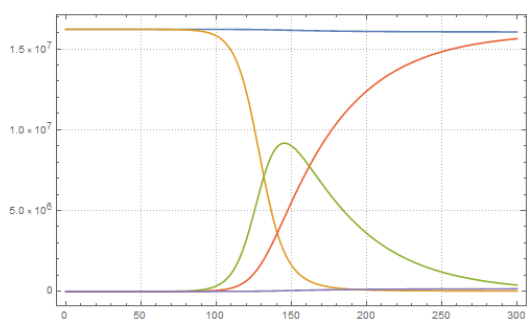

(d) Plot of the SIR model $\sqrt{2}$ with the parameters $\beta=0.15, \gamma=1 / 45$ and the proportion of death $\mu=0.01 \gamma$. And plot of the fit without data.

Figure 7: Plot of the SIR model(2) compared with plot of Senegal's data in table 1. The total population of Senegal we use is $N=1674392 \%$. On the abscissa axis, the graduation 40 represents 2020, March 31. 
medRxiv preprint doi: https://doi.org/10.1101/2020.04.26.20081042; this version posted May 1, 2020. The copyright holder for this preprint (which was not certified by peer review) is the author/funder, who has granted medRxiv a license to display the preprint in perpetuity.

It is made available under a CC-BY 4.0 International license .

SIR model fit to COVID-19 data 2020 , April $\bullet$ Vol. , No.

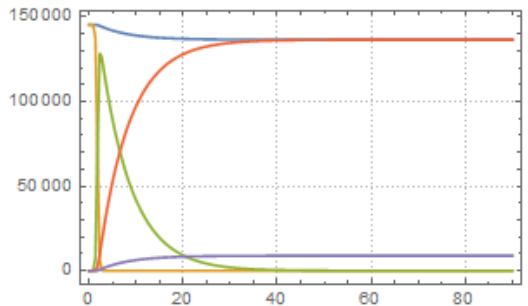

(a) Plot of the SIR model 2 with the parameters $\beta=5.0753$ and $\gamma=1 / 45$ and the proportion of death $\mu=$ $0.11 \gamma$. The susceptible $S(t)$ in red line, The population in blue line, the infected $I(t)$ in green line, the recovered in orange line and the death $D(t)$ in purple. The total population is reduced.

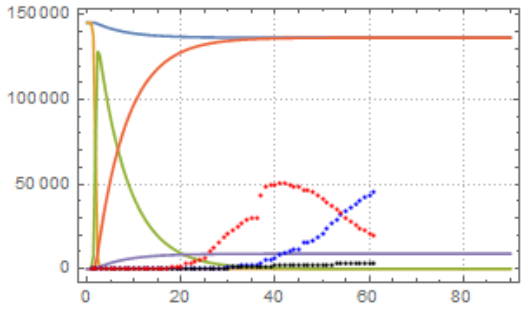

(b) Plot of the SIR model 2 with the parameters $\beta=5.0753, \gamma=1 / 45$, the proportion of death $\mu=0.01 \gamma$ and reduced population. The susceptible $S(t)$ in red line, The population in blue line, the infected $I(t)$ in green line, the recovered in orange line and the death $D(t)$ in purple line. And plot without fit to the Infected data in red dotted, the recovered data in blue dotted, the death data in black dotted.

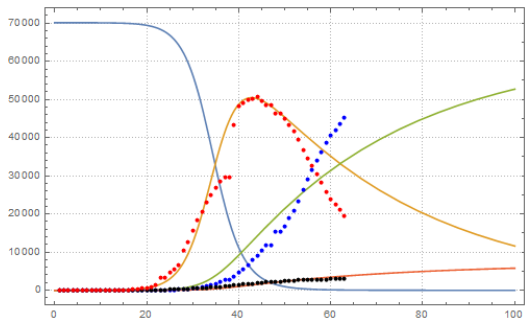

(c) Plot of the SIR model 2 with the parameters $\beta=0.35, \gamma=1 / 40$ and the proportion of death $\mu=0.11 \gamma$. And plot with fit to the Infected data in red dotted, the recovered data in blue dotted, the death data in black dotted.

Figure 8: Plot of the SIR model(2) compared with plot of China's data in table 2. The total population of Hubei we use is $N=59172000$ (https://en.wikipedia.org/wiki/2020_Hubei_lockdowns). On the abscissa axis, the graduation 63 represents 2020, March 08. 


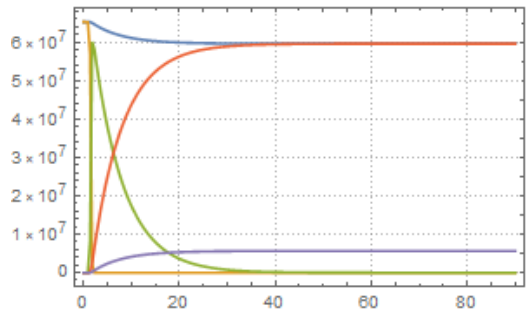

(a) Plot of the SIR model 2 with the parameters $\beta=5.0753$ and $\gamma=1 / 45$ and the proportion of death $\mu=$ $0.01 \gamma$. The susceptible $S(t)$ in red line, The population in blue line, the infected $I(t)$ in green line, the recovered in orange line and the death $D(t)$ in purple.

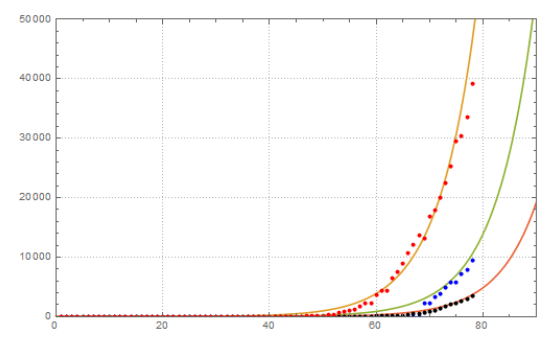

(c) Plot of the SIR model 2 with the parameters $\beta=0.18, \gamma=1 / 32$ and the proportion of death $\mu=0.01 \gamma$. And plot with fit to data.

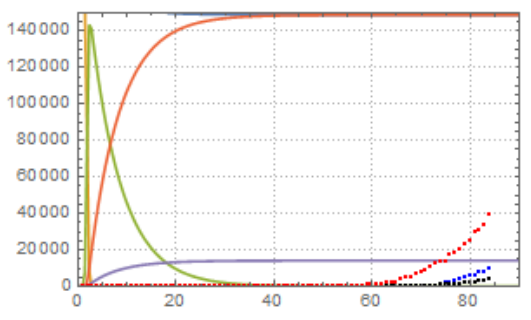

(b) Plot of the SIR model 2 with the parameters $\beta=5.0753, \gamma=1 / 45$, the proportion of death $\mu=0.01 \gamma$ and reduced population. And plot without fit to the Infected data in red dotted, the recovered data in blue dotted, the death data in black dotted.

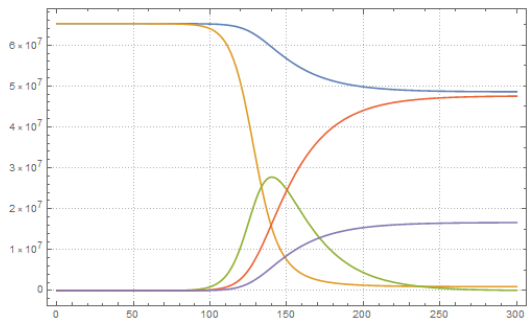

(d) Plot of the SIR model 2 with the parameters $\beta=0.18, \gamma=1 / 32$ and the proportion of death $\mu=0.01 \gamma$. And plot of the fit without data.

Figure 9: Plot of the SIR model(2) compared with plot of France's data in table 3 . The total population of France we use is $N=65241903$. On the abscissa axis, the graduation 63 represents 2020, March 31.

\section{v. Discussion}

The SIR model is fitted to the data. This allows us to note that the maximum number of infected can go up to 900000 (figure 7d) in Senegal and 2700000 (figure 9d) in France. While in the scaling the figures $6 \mathrm{a}$ shows that the maximum number of infected can go up to 320000 . However, this analysis does not take into account the nationwide anti-pandemic measures.

Using the results shown in the figures $2 \mathrm{c}, 2 \mathrm{e}, 2 \mathrm{~d}$, 2f, $4 \mathrm{a}, 4 \mathrm{~b}, 5 \mathrm{a}$ and $5 \mathrm{~b}$, we can see that the data deviate from their first exponential evolution to follow a new, slower trajectory which can still be exponential. To understand the nature of this new trajectory, the way to manage the contact rate is decisive and can lead to different analyzes. However, having considered a slow decrease over time of the contact rate, we can see in the figures $2 \mathrm{e}, 2 \mathrm{f}, 4 \mathrm{~b}$ and $5 \mathrm{~b}$ that the updated data pass under the new trajectory for Senegal while in France the data follow the new trajectory. This could, therefore, be due to the effects of the measures taken by these countries. 


\section{PREDICTION}

In this section, we plot some predictions for Senegal. The prediction does not concern the total confirmed cases, but only the effective cases obtained by reduced from the total confirmed cases, the recovered cases and the death cases.

We show the curve of effective infected cases given by $T N I(t)-R(t)-D(t)$ with both $\tilde{\beta}(4)$ and (5), the curve of the fitted infected $I(t)$ in figure $7 \mathrm{c}$ and the curve obtained by using machine learning. We plot these curves with additional dates until 2020, April 21.

We use machine learning, based only on data, to do forecasting. "Predict" is a function of "Automated Machine Learning" in Wolfram Mathematica. It allows for automatic training and data prediction. We can choose different method of regression algorithm: "RandomForest", "LinearRegression", "NeuralNetwork", "GaussianProcess", "NearestNeighbors", etc. We use the "NeuralNetwork" regression algorithm which predicts using an artificial neural network.

Let's recall that our forecasting use in a first part the results of the works in subsection iii and iii where we use a SIR model to fit data. And in a second part, the forecasting using only data.

The forecasting show in figures $10 \mathrm{a}, 10 \mathrm{c}$ an optimistic situation for Senegal. Also the machine learning figure $10 \mathrm{e}$ shows an optimistic forecasting. But in all cases, we see that the additional data (March 31-06) goes down the predicting path. It may be caused by the nationwide antipandemic measures in Senegal. In addition, we have considered in figures 10a and 10b a contact rate gradually reduced since the measures were taken and we see that the data come below the path of the predicted curve. 
SIR model fit to COVID-19 data 2020 , April $\bullet$ Vol. , No.

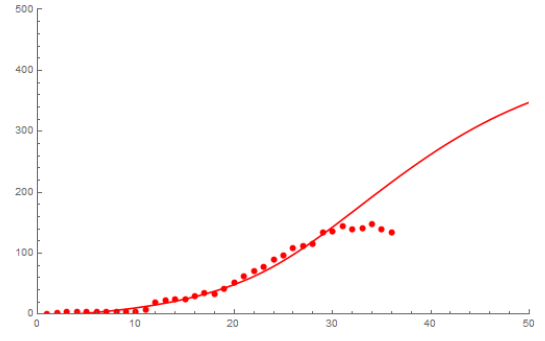

(a) Plot of the number of infected cases using the scaling SIR model 6 with $\tilde{\beta}$ given by (4). On the abscissa axis, the graduation 30 represents 2020 , March 31. Then 2020, April 06 is the graduation 36 .

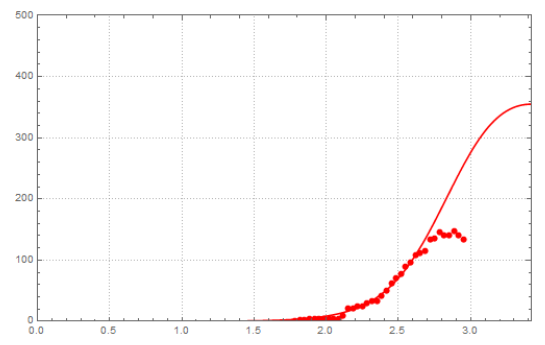

(c) Plot of the number of infected cases using the scaling SIR model (8). On the abscissa axis, the graduation 2.8 represents 2020, March 31 . Then 2020, April 06 is the graduation 3.0.

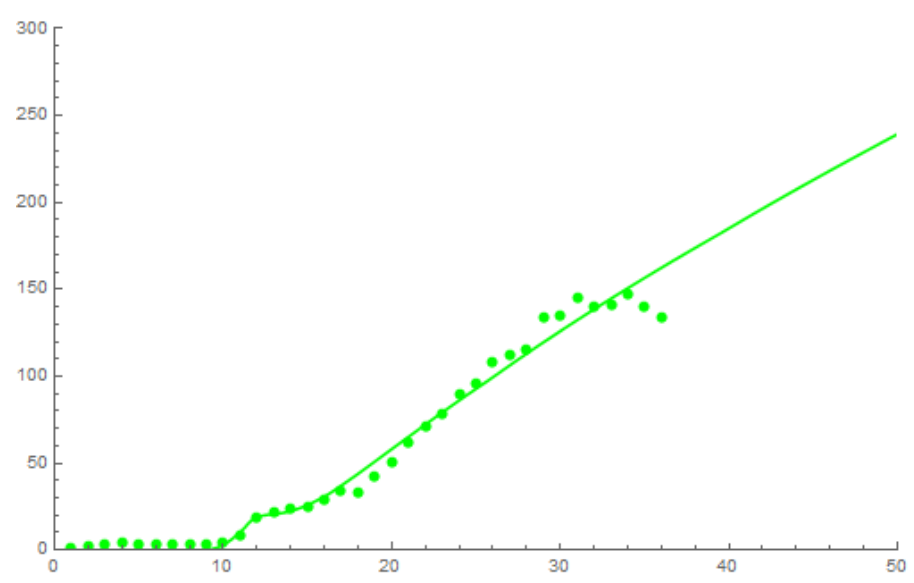

(e) Plot of the number of infected cases by training a PredictorFunction to predict the average next values of infected. On the abscissa axis, the graduation 30 represents 2020, March 31. Then 2020, April 06 is the graduation 36.

Figure 10: Forecasting for Senegal, using the results of the works in subsection $i$ ii, iii and machine learning. The data plotted (red dotted, green dotted), is given in table 1 , but completed until 2020, April 06.

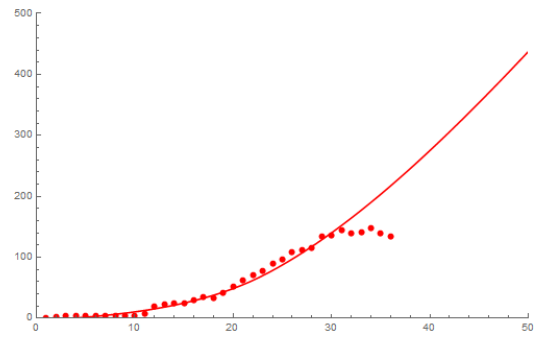

(b) Plot of the number of infected cases using the scaling SIR model $\sqrt{6}$ with $\tilde{\beta}$ given by 5. On the abscissa axis, the graduation 30 represents 2020 , March 31. Then 2020, April 06 is the graduation 36 .

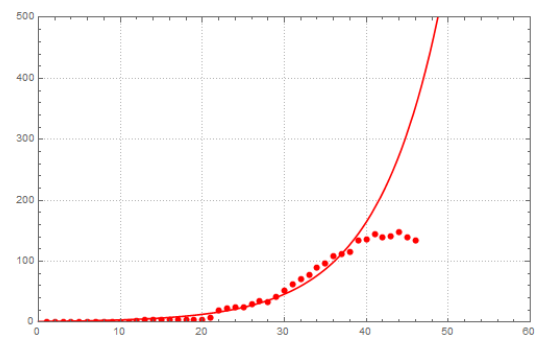

(d) Plot of the number of infected cases using SIR model (2) with parametric solve. On the abscissa axis, the graduation 40 represents 2020 , March 31. Then 2020, April 06 is the graduation 46 . 


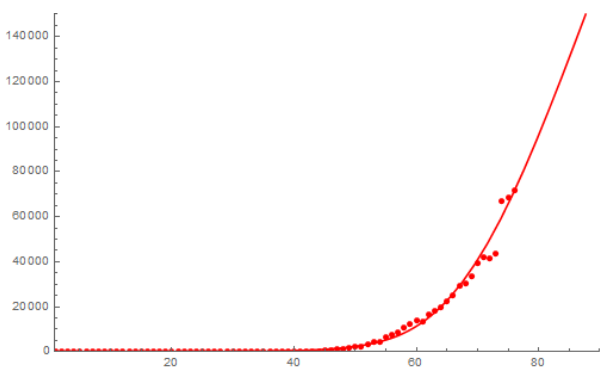

(a) Plot of the number of infected cases using the scaling SIR model 6 with $\tilde{\beta}$ given by (4). On the abscissa axis, the graduation 70 represents 2020, March 31 . Then 2020 , April 06 is the graduation 76 .

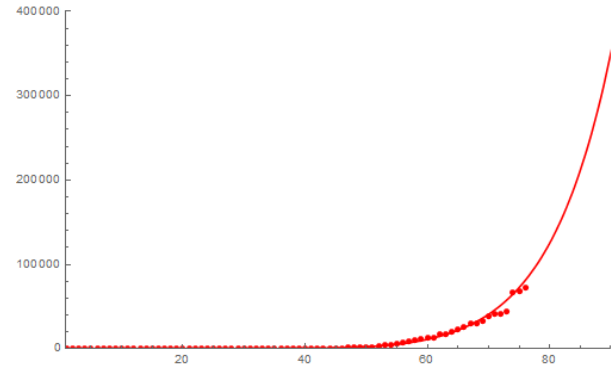

(b) Plot of the number of infected cases using the scaling SIR model (6) with $\tilde{\beta}$ given by [5. On the abscissa axis, the graduation 70 represents 2020, March 31. Then 2020, April 06 is the graduation 76 .

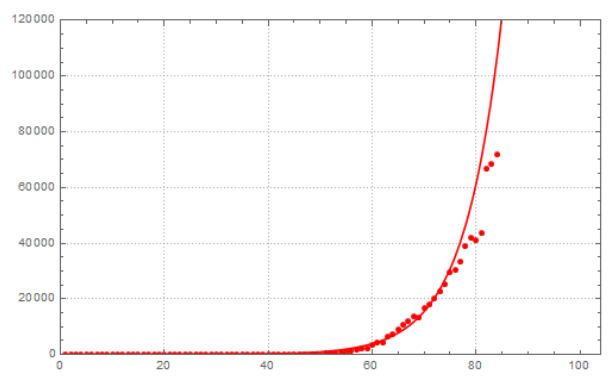

(c) Plot of the number of infected cases using SIR model 22 with parametric solve. On the abscissa axis, the graduation 70 represents 2020, March 31. Then 2020, April 06 is the graduation 76 .

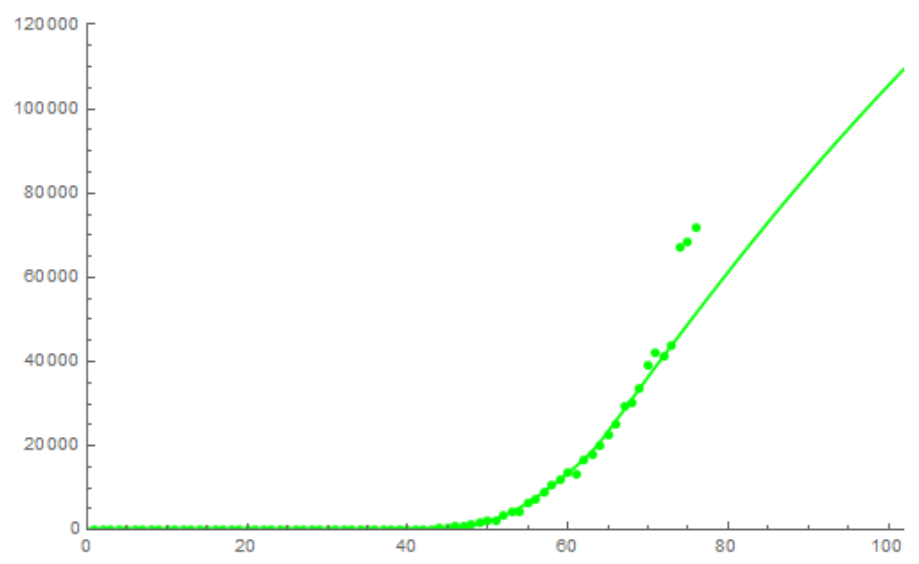

(d) Plot of the number of infected cases by training a PredictorFunction to predict the average next values of infected. On the abscissa axis, the graduation 70 represents 2020, March 31. Then 2020, April 06 is the graduation 76 .

Figure 11: Forecasting for France, using the results of the works in subsection $i$ ii and iii and machine learning. The data plotted (red dotted, green dotted), is given in table 3 , but completed until 2020, April 06. 


\section{Conclusion And Perspectives}

In this paper, we have used the classical SIR model for fitting data and then we have done forecasting. We have also estimated the contact rate, and the average infectious period parameters of the SIR model to obtain fit. Machine learning can help in epidemiology to understand the disease, but also to study the impact or effectiveness of the anti-pandemic measures taken.

We can also study the ideal date for stopping the containment measures. Ideal in the sense that even without the measures the disease can no longer spread. We can also study the possibility of making periodic confinements to reduce the economic impact of the measures. The economy may be impacted by the evolution of the disease and measures. It would, therefore, be interesting to couple economic models with epidemiological models. Since the impact can also be long-term, scaling can be used to dimension the epidemiological-economic coupling. The impact of the environment is also to be taken into account in the models. This means studying the contamination due to the environment (air, objects, etc).

\section{APPENDIX}

\section{i. Using function fit in SIR model}

From $T N I(t)=b \exp (c t)-a$, we have $T N I\left(t_{0}\right)=b \exp \left(c t_{0}\right)-a$ and using (3), we obtain

$$
b \exp \left(c t_{0}\right)-a=0 .
$$

We deduce $a=b \exp \left(c t_{0}\right)$, then $t_{0}=\frac{\ln (a)-\ln (b)}{c}$.

Again from (3) we have

$$
I(t)=T \dot{N} I(t)=b c \exp (c t)
$$

Then $I\left(t_{0}\right)=b c \exp \left(c t_{0}\right)=a c=I_{0}$ and $\frac{I(t)}{I\left(t_{0}\right)}=\exp \left(c\left(t-t_{0}\right)\right)$. Hence, we obtain

$$
I(t)=I\left(t_{0}\right) \exp \left(c\left(t-t_{0}\right)\right),
$$

then $\dot{I}\left(t_{0}\right)=c I\left(t_{0}\right)$.

From the second equation in the SIR model (1), we obtain at $t_{0}, \dot{I}\left(t_{0}\right)=\frac{\beta I_{0} S_{0}}{N}-\gamma I_{0}=c I\left(t_{0}\right)$. Hence, we get

$$
c=\frac{\beta S_{0}}{N}-\gamma .
$$

Using the third equation in the SIR model (1) yields

$$
\dot{R}(t)=\gamma I\left(t_{0}\right) \exp \left(c\left(t-t_{0}\right)\right),
$$

then integrating, we obtain

$$
R(t)=\frac{\gamma}{c}\left(I(t)-I\left(t_{0}\right)\right) .
$$




\section{ii. Determination of the scaled SIR}

$S(t)=S\left(t_{c} \bar{t}\right), I(t)=I\left(t_{c} \bar{t}\right)$ and $R(t)=R\left(t_{c} \bar{t}\right)$, then replacing in left members of the SIR model (1), we obtain:

$$
\begin{gathered}
\dot{S}=\frac{S_{c}}{t_{c}} \dot{\bar{S}} \\
\dot{I}=\frac{I_{c}}{t_{c}} \dot{\bar{I}} \\
\dot{R}=\frac{R_{c}}{t_{c}} \dot{\bar{R}}
\end{gathered}
$$

Replacing again in right members of the SIR model (1), we get:

$$
\begin{aligned}
\dot{S} & =-\frac{\beta S_{c} I_{c}}{N} \bar{I} \bar{S} \\
\dot{I} & =\frac{\beta S_{c} I_{c}}{N} \bar{I} \bar{S}-\gamma I_{c} \bar{I} \\
\dot{R} & =\gamma I_{c} \bar{I}
\end{aligned}
$$

Then we have:

$$
\begin{aligned}
& \frac{S_{c}}{t_{c}} \dot{\bar{S}}=-\frac{\beta S_{c} I_{c}}{N} \bar{I} \bar{S} \\
& \frac{I_{c}}{t_{c}} \dot{\bar{I}}=\frac{\beta S_{c} I_{c}}{N} \bar{I} \bar{S}-\gamma I_{c} \bar{I} \\
& \frac{R_{c}}{t_{c}} \dot{\bar{R}}=\gamma I_{c} \bar{I}
\end{aligned}
$$

Finally, we obtain:

$$
\left\{\begin{array}{l}
\dot{\bar{S}}=-\frac{\beta I_{c} t_{c}}{N} \bar{I} \bar{S} \\
\dot{\bar{I}}=\frac{\beta S_{c} t_{c}}{N} \bar{I} \bar{S}-\gamma t_{c} \bar{I} \\
\dot{\bar{R}}=\gamma \frac{I_{c} t_{c}}{R_{c}} \bar{I}
\end{array}\right.
$$

\section{REFERENCES}

[Alpaydin, 2010] E. Alpaydin. (2010). Introduction to Machine Learning. 2nd ed. Massachusetts Institute of Technology, ISBN 978-0-262-01243-0.

[Anderson and May, 1991] R. M. Anderson and R. M. May. (1991). Infectious Diseases of Humans.m Oxford University Press, Oxford.

[Antonov CPMC, 2020] A. Antonov. (2020). Coronavirus propagation modeling considerations. SystemModeling at GitHub, https://github.com/antononcube/SystemModeling.

[Antonov EMMP, 2020] A. Antonov. (2020). Epidemiology models Mathematica package. SystemModeling at GitHub, https://github.com/antononcube/SystemModeling. 
[Antonov EMMMP, 2020] A. Antonov. (2020). Epidemiology models modifications Mathematica package. SystemModeling at GitHub, https://github.com/antononcube/SystemModeling.

[Antonov SDIIMP, 2020] A. Antonov. (2020). System dynamics interactive interfaces functions Mathematica package. SystemModeling at GitHub, https://github.com/antononcube/SystemModeling.

[Hethcote, 2000] H. W. Hethcote. (2000). The Mathematics of Infectious Diseases. Society for Industrial and Applied Mathematics, Vol. 42, No. 4, pp. 599-653.

[Langtangen and Pedersen, 2016] H. P. Langtangen and G. K. Pedersen. (2009). Scaling of Differential Equations. Springer.

[Lauro, Kiss and Miller, 2020] F. Di Lauro, I.Z. Kissy, and J.C. Miller. (2020). The timing of one-shot interventions for epidemic control. medRxiv preprint doi: https://doi.org/10.1101/2020.03.02.20030007.

[Liu, Magal, Seydi and Webb, 2020] Z. Liu, P. Magal,O. Seydi, G. Webb, (2020). Understanding Unreported Cases in the 2019-Ncov Epidemic Outbreak in Wuhan, China, and the Importance of Major Public Health Interventions. SSRN: https://ssrn.com/abstract=3530969 or http://dx.doi.org/10.2139/ssrn.3530969.

[Liu, Magal, Seydi and Webb, 2020] Z. Liu, P. Magal,O. Seydi, G. Webb, (2020). Predicting the cumulative number of cases for the COVID-19 epidemic in China from early data. medRxiv preprint https://doi.org/10.1101/2020.03.11.20034314.

[Wolfram Research] Wolfram Research. Epidemic Data for Novel Coronavirus COVID-19. WolframCloud, https://www.wolframcloud.com/. 